\title{
A Photometricity and Extinction Monitor at the Apache Point Observatory
}

\section{Citation}

Hogg, David W., Douglas P. Finkbeiner, David J. Schlegel, and James E. Gunn. 2001. “A Photometricity and Extinction Monitor at the Apache Point Observatory." The Astronomical Journal 122 (4) (October): 2129-2138. doi:10.1086/323103.

\section{Published Version}

$10.1086 / 323103$

\section{Permanent link}

http://nrs.harvard.edu/urn-3:HUL.InstRepos:33461897

\section{Terms of Use}

This article was downloaded from Harvard University's DASH repository, and is made available under the terms and conditions applicable to Other Posted Material, as set forth at http:// nrs.harvard.edu/urn-3:HUL.InstRepos:dash.current.terms-of-use\#LAA

\section{Share Your Story}

The Harvard community has made this article openly available.

Please share how this access benefits you. Submit a story.

Accessibility 
The Astronomical Journal, 122:2129-2138, 2001 October

\title{
A PHOTOMETRICITY AND EXTINCTION MONITOR AT THE APACHE POINT OBSERVATORY ${ }^{1}$
}

\author{
DAVID W. HoGG ${ }^{2}$ \\ Department of Physics, New York University, 4 Washington Place, New York, NY 10003; david.hogg@nyu.edu \\ AND \\ Douglas P. Finkbeiner, ${ }^{3,4}$ David J. Schlegel, and James E. GunN \\ Department of Astrophysics, Peyon Hall, Ivy Lane, Princeton University, Princeton, NJ 08544; dfink@astro.princeton.edu, \\ schlegel@astro.princeton.edu, jeg@astro.princeton.edu \\ Received 2001 May 23; accepted 2001 June 25
}

\begin{abstract}
An unsupervised software "robot" that automatically and robustly reduces and analyzes CCD observations of photometric standard stars is described. The robot measures extinction coefficients and other photometric parameters in real time and, more carefully, on the next day. It also reduces and analyzes data from an all-sky $10 \mu \mathrm{m}$ camera to detect clouds; photometric data taken during cloudy periods are automatically rejected. The robot reports its findings to observers and data analysts via the World Wide Web. It can be used to assess photometricity and to build data on site conditions. The robot's automated and uniform site monitoring represents a minimum standard for any observing site with queue scheduling, a public data archive, or likely participation in any future National Virtual Observatory.

Key words: astrometry - methods: data analysis — methods: observational — surveys techniques: image processing — techniques: photometric
\end{abstract}

\section{INTRODUCTION}

More and more, astronomical research is being performed remotely, in the sense that the observer, or perhaps more properly "data analyst," is now often not present at the place or time at which observations are taken. The increase in remoteness has several causes. One is that for many observatories, telecommunication is easier than travel, especially if telescope allocations are of short duration. Another is that several new telescopes are using or plan to use queue scheduling (e.g., contributions to Boroson, Davies, \& Robson 1996 and Boroson et al. 1998; Garzon \& Rozas 1998; Tilanus 2000; Massey, Guerrieri, \& Joyce 2000), for which observer travel is essentially impossible. Some new ground-based telescopes are partially or completely robotic (e.g., contributions to Filippenko 1992; contributions to Adelman, Dukes, \& Adelman 1992; also Baruch \& da Luz Vieira 1993; Akerlof et al. 1999; CastroTirado et al. 1999; Strassmeier et al. 2000; Querci \& Querci 2000). Possibly the most important reason for the increase in remote observing is that many observatories, many large surveys, and some independent organizations are creating huge public data archives that allow analyses by anyone at any time (e.g., contributions to Mehringer, Plante, \& Roberts 1999 and Manset, Veillet, \& Crabtree 2000). There has been community discussion of a "National Virtual Observatory," which might be a superset of these archives and surveys (e.g., contributions to Brunner, Djorgovski, \& Szalay 2001).

Remote observing and archival data analysis bring huge economic and scientific benefits to astronomy but with the

\footnotetext{
${ }^{1}$ Based on observations obtained at the Apache Point Observatory, which is owned and operated by the Astrophysical Research Consortium.

${ }^{2}$ Also Institute for Advanced Study, Olden Lane, Princeton, NJ 085400631.

${ }^{3}$ Also Department of Astronomy, University of California at Berkeley, 601 Campbell Hall, Berkeley, CA 94720.

${ }^{4}$ Hubble Fellow.
}

significant cost that the observer does not have direct access to observing conditions at the site. Most remote observatories and data archives keep logs written by telescope operators, but these logs are notoriously nonuniform in their attention to detail and use of terminology. All sites that plan to host remote observers or maintain public data archives must have repeatable, quantitative, astronomically relevant site monitoring.

For these reasons, among others (involving photometric calibration and direction of survey operations) the Sloan Digital Sky Survey (SDSS; York et al. 2000), which is constructing a public database of $10^{4} \mathrm{deg}^{2}$ of five-bandpass optical imaging and $10^{6}$ optical spectra, employs several pieces of hardware for monitoring the Apache Point Observatory site, including a $10 \mu \mathrm{m}$ cloud-camera scanning the whole sky (Hull, Limmongkol, \& Siegmund 1994), a singlestar atmospheric seeing monitor, a low-altitude dust particle counter, a basic weather station, and a $0.5 \mathrm{~m}$ telescope making photometric measurements of a large set of standard stars. This paper is about a fully automated software "robot" that reduces the raw $0.5 \mathrm{~m}$ telescope data, locates and measures standard stars, and determines atmospheric extinction in near-real time, reporting its findings to telescope and survey operators via the World Wide $\mathrm{Web}^{5}$ (WWW). This paper describes the software robot, rather than the hardware, which will be the subject of a separate paper (Uomoto et al. 2001).

Although the robot is somewhat specialized to work with the hardware and data available at the Apache Point Observatory, it could be generalized easily for different hardware. We are presenting it here because it might serve as a prototype for site monitors that ought to be part of any functional remote observing site and of the National Virtual Observatory.

\footnotetext{
${ }^{5}$ Information about the WWW is available at http://www.w3.org/.
} 
TABLE 1

APPROXIMATE PT BANDPASS INFORMATION

\begin{tabular}{crrcrr}
\hline \hline Parameter & \multicolumn{1}{c}{$u^{\prime}$} & \multicolumn{1}{c}{$g^{\prime}$} & \multicolumn{1}{c}{$r^{\prime}$} & \multicolumn{1}{c}{$i^{\prime}$} & \multicolumn{1}{c}{$z^{\prime}$} \\
\hline Central wavelength $(\AA) \ldots \ldots$. & 3540 & 4770 & 6230 & 7620 & 9130 \\
FWHM $(\AA)$.................. & 570 & 1390 & 1370 & 1530 & 950 \\
\hline
\end{tabular}

\section{TELESCOPE AND DETECTOR}

The primary telescope used in this study is the Photometric Telescope (PT) of the SDSS, located at the Apache Point Observatory (APO) in New Mexico, at latitude $32^{\circ} 46^{\prime} 49^{\mathrm{s}} 30 \mathrm{~N}$, longitude $105^{\circ} 49^{\prime} 13.50 \mathrm{~W}$, and elevation $2788 \mathrm{~m}$. The PT has a 20 inch $(0.5 \mathrm{~m})$ primary mirror and is outfitted with a $2048 \times 2048$ pixel CCD with 1".16 pixels, making for a $40 \times 40 \mathrm{arcmin}^{2}$ field of view. The telescope and CCD will be described in more detail elsewhere (Uomoto et al. 2001).

The telescope takes images through five bandpasses, $u^{\prime}$, $g^{\prime}, r^{\prime}, i^{\prime}$, and $z^{\prime}$, chosen to be close to those in the SDSS $2.5 \mathrm{~m}$ imaging camera. Filter wavelengths are given in Table 1. The magnitude system here is based on an approximation to an AB system (Oke \& Gunn 1983; Fukugita et al. 1996), again because that was the choice for the SDSS imaging. The photometric system will be described in more detail elsewhere (Smith et al. 2001). Nothing about the function of the robot is tied to this photometric system; any system can be used provided that there is a well-calibrated network of standard stars.

\section{OBSERVING STRATEGY}

Site monitoring and measurement of atmospheric extinction is only one part of the function of the PT. The PT is being simultaneously used to take data on a very large number of secondary patches that will provide calibration information for the SDSS imaging. For this reason, the PT spends only about one-third of its time monitoring standard stars. Its site monitoring and atmospheric extinction measuring functions could be improved significantly, in signal-to-noise and time resolution, if the PT were dedicated to these tasks.

The observing plan for the night is generated automatically by a field "autopicker, " which chooses standard-star fields on the basis of (1) observability, (2) air mass coverage, (3) intrinsic stellar color coverage, and (4) number of calibrated stars per standard field. Because the observing is very regular, with $u^{\prime}, g^{\prime}, r^{\prime}, i^{\prime}$, and $z^{\prime}$ images taken (in that order) of each field, it has been almost entirely automated. The only significant variation in observing from standard field to standard field is that different fields are imaged for different exposure times to avoid saturation.

The raw data from the telescope is in the form of images in the Flexible Image Transport System format (FITS; Wells, Greisen, \& Harten 1981). The raw image headers contain the filter (bandpass) used, the exposure time, the date and UT at which the exposure was taken, the approximate pointing of the telescope in right ascension and declination, and the type of exposure (e.g., bias, flat, standard-star field, or secondary patch). The robot makes use of much of this information, as described below.

\section{OBTAINING AND MANIPULATING THE DATA}

In principle, the photometricity monitoring software could run on the data acquisition computer. However, in the interest of limiting stress on the real-time systems, the photometricity robot runs on a separate machine, obtaining its data by periodic executions of the UNIX RSYNC software. ${ }^{6}$ The RSYNC software performs file transfer across a network, using a remote-update protocol (based on file check sums) to ensure that it transfers only updated or new files, without duplicating effort. The output of RSYNC can be set to include a list of the files that were updated. This output is passed (via scripts written in the UNIX shell language $\mathrm{BASH}^{7}$ ) to a set of software tools written in the data analysis language IDL. ${ }^{8}$

Virtually all the image processing, data analysis, fitting, and feedback to the observer is executed with IDL programs in BASH wrappers. This combination of software has proven to be very stable and robust over many months of continuous operation. In addition, data reduction code written in IDL is easy to develop and read. Our only significant reservation is that IDL is a commercial product, which is not open source. We have responded to this lack of transparency by writing as much as possible of the robot's function in terms of very low level IDL primitives, which could be rewritten straightforwardly in any other data analysis language if we lost confidence in or lost access to IDL. We have not found the lack of transparency to be limiting for any of the functionality described in this paper.

\section{CORRECTING THE IMAGES}

Each raw image is bias-subtracted and flattened, using biases and flat-field information archived from the most recent photometric night. How the bias and flat images are computed from a night's data is described in $\S 11$, along with the conditions on which a night is declared photometric.

Because the CCD is thinned for sensitivity in the $u$ bandpass, it exhibits interference fringing in the $i^{\prime}$ and $z^{\prime}$ bandpasses. The fringing pattern is very stable during a night and from night to night. The fringing is modeled as an additive distortion; it is removed by subtracting a "fringe image" scaled by the DC sky level. How the fringe image is computed is described in $\S 11$. The DC sky level is estimated by taking a mean of all pixels in the image, but with iterated " $\sigma$ clipping, " in which the rms pixel value is computed, 3.5 $\sigma$ outlier pixels are discarded, and the mean and rms are reestimated. This process is iterated to convergence. The fringing correction is demonstrated in Figure 1. Although in principle the fringing pattern may depend on the temperature or humidity of the night sky, it does not appear to vary significantly within a night, or even night to night. Perhaps surprisingly, the fringing pattern appears fixed and its amplitude scales linearly with the DC level of the sky.

For each corrected PT image, a mask of bad pixels is retained. The mask is the union of all pixels saturated in the raw images, along with all pixels that are marked as anomalous in the bias image, flat image, or (for the $i^{\prime}$ and $z^{\prime}$ bandpasses) fringe image. How pixels are marked as anomalous in the bias, flat, and fringe is described in $\S 11$.

The individual bias-subtracted, flattened, and (for the $i^{\prime}$ and $z^{\prime}$ bandpasses) fringe-corrected images will hereafter be

\footnotetext{
${ }^{6}$ Information about RSYNC is available at http://rsync.samba.org/.

${ }^{7}$ Information about BASH is available at the Free Software Foundation at http://www.fsf.org/.

${ }^{8}$ Information about IDL is available at http://www.rsinc.com/.
} 

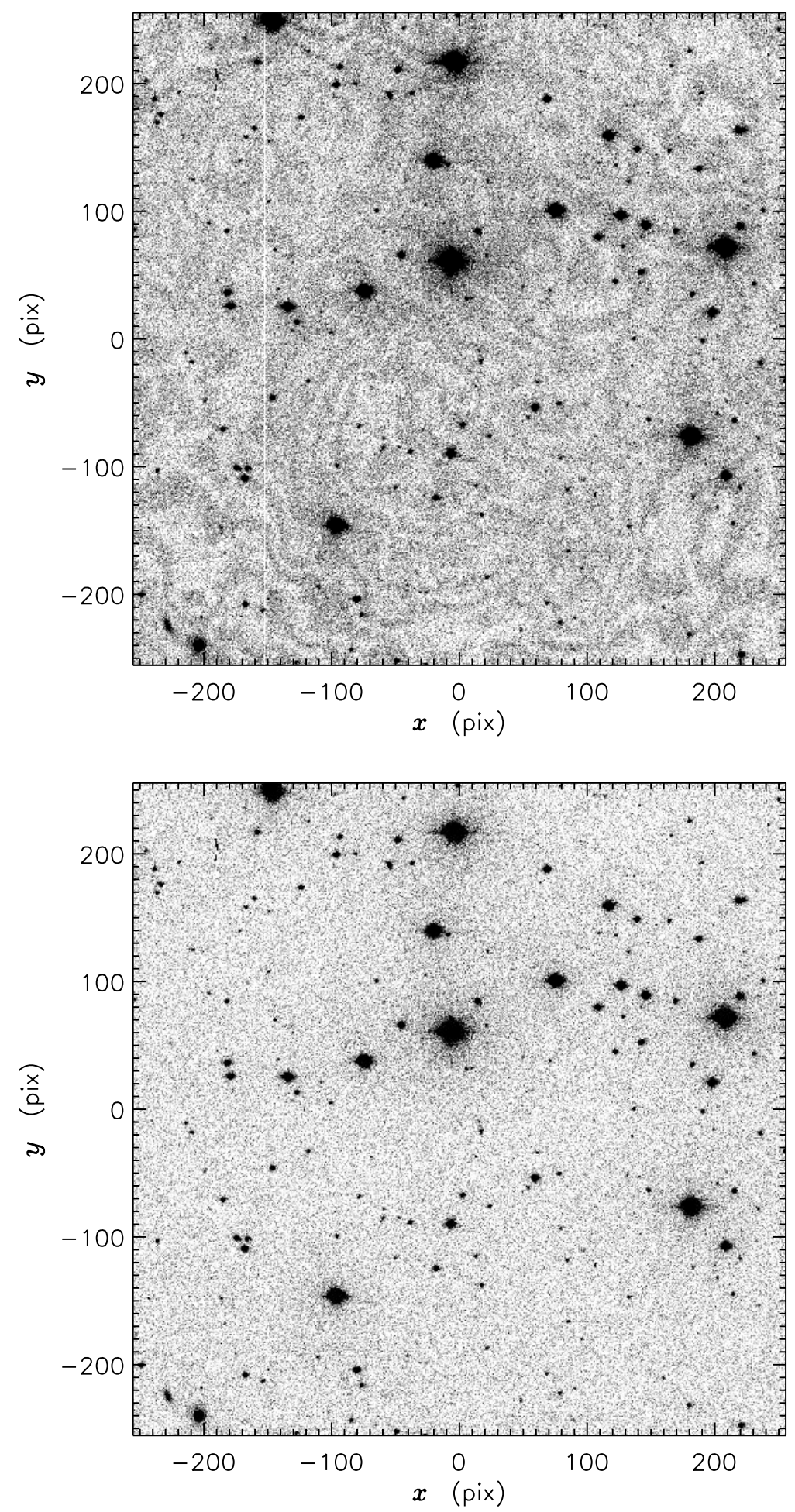

FIG. 1 . $-512 \times 512 \operatorname{pixel}^{2}\left(594 \times 594 \operatorname{arcsec}^{2}\right)$ section of a bias-subtracted and flat-fielded $z^{\prime}$-bandpass image from the PT before (top) and after (bottom) the fringing correction. The two images are stretched identically; in the top image, the fringing is roughly $10 \%$ of the background level, peak to peak.

referred to as the "corrected frames." The corrected frames are stored and saved by the robot in FITS format.

\section{ASTROMETRY AND SOURCE IDENTIFICATION}

Hardware pointing precision is not adequate for individual source identifications. The software robot determines the precise astrometry itself, by comparison with the USNO-SA2.0 astrometric catalog (Monet et al. 1998). This catalog contains $5 \times 10^{7}$ astrometric stars over most of the sky; there are typically a few times $10^{2}$ catalog stars inside a PT image.

In brief, the astrometric solution for each image is found as follows: A subcatalog is created from all astrometric stars within $30^{\prime}$ of the field center (as reported by the hardware in the raw image header). An implementation of the DAOPHOT software (Stetson 1987, 1992) in IDL (W. 
Landsman 2000, private communication) is used to locate all bright point sources in the corrected frame (DAOPHOT is used for object finding only, not photometry). Each set of stars is treated as a set of delta functions on the twodimensional plane of the sky, and the cross-correlation image is constructed. If there is a statistically significant cluster of points in the cross-correlation image, it is treated as the offset between the two images. Corresponding positions in the two sets of stars (from the corrected frame and from the astrometric catalog) are identified, and the offset, rotation, and nonlinear radial distortion in the corrected frame are all fitted, with iterative improvement of the correspondence between the two sets of stars. The precise astrometric information is stored in the FITS header of each corrected frame in GSSS format (this format is not standard FITS but is used by the HST Guide Star Survey; e.g., Russell et al. 1990; Calabretta \& Greisen 2000). Our algorithm is much faster, albeit less general, than previous algorithms (e.g., Valdes et al. 1995).

Essentially all exposures of more than a few seconds in the $g^{\prime}, r^{\prime}$, and $i^{\prime}$ bandpasses obtain correct astrometric solutions by this procedure. Some short $u^{\prime}$ and $z^{\prime}$ exposures do not and are picked up on a second pass using a minicatalog constructed from stars detected in the $g^{\prime}$ and $r^{\prime}$ exposures of the same field. On most nights, all exposures in all bands obtain correct astrometric solutions. The algorithm and its implementation will be discussed in more detail in a separate paper, as it has applications in astronomy that go beyond this project.

\section{PHOTOMETRY}

The robot measures and performs photometric fits with the stars in the SDSS catalog of photometric standards (Smith et al. 2001). This catalog includes stars in the range 6 mag $<r<15$ mag, calibrated with the USNO 40 inch $(1 \mathrm{~m})$ Ritchey-Chrétien telescope. Several of the standard-star fields used in this catalog are well-studied fields (Landolt 1992) containing multiple standard stars spanning a range of magnitude and color.

The photometric catalog is searched for photometric standard stars inside the boundaries of each corrected frame with precise astrometric header information. If any stars are found in the photometric catalog, aperture photometry is performed on the point source found at the astrometric location of each photometric standard star. The centers of the photometric apertures are tweaked with a centroiding procedure that allows for small $\left(\lesssim 1^{\prime \prime}\right)$ inaccuracies in absolute astrometry. The aperture photometry is performed in focal plane apertures of radii $4.63,7.43$, and 11".42. The sky value is measured by taking the mean of the pixel values in an annulus of inner radius 28 ".20 and outer radius 44 ".21, with iterated outlier rejection at $3 \sigma$. All these angular radii are chosen to match those used by the SDSS PHOTO software (Lupton et al. 2001a, 2001b).

In what follows, the 7".43 radius aperture photometry is used. This aperture was chosen from among the three for showing on nights of typical seeing the lowest-scatter photometric solutions. This is because, in practice, the 7".43 radius aperture is roughly the correct trade-off between individual measurement signal-to-noise (which favors small apertures) and insensitivity to spatial or temporal variations in the point-spread function (which favors large ones). The PT shows significant, repeatable, systematic distortions of the point-spread function across the field of view; a more sophisticated robot would model and correct these distortions; for our purposes it is sufficient to simply choose the relatively large 7.43 radius aperture.

Each photometric measurement is corrected for its location in the field of view of the PT. There are two corrections. The first is an illumination correction derived from the radial distortion of the field as found in the precise astrometric solution (\$6). The illumination correction is designed to account for the fact that photometrically we are interested in fluxes, but the flat field is measured with the sky; i.e., the flat field is made to correct pixels to a constant surface brightness sensitivity rather than a constant flux sensitivity. Because of optical distortions, pixels near the edge of the CCD see a different solid angle than pixels near the center. Empirically, the dominant field distortion appears radial, so no attempt has been made to correct for illumination variation from arbitrary distortions. The illumination correction reaches a maximum of $\sim 0.02 \mathrm{mag}$ at the field edges and $\sim 0.04 \mathrm{mag}$ at the field corners.

The second correction is related to the fringing in the $i^{\prime}$ and $z^{\prime}$ bandpasses. The CCD is thinned, but not precisely uniformly; the dominant thinning gradients are radial. Because of reflections internal to the $\mathrm{CCD}$, gradients in CCD thickness lead to gradients in the fraction of pointsource light scattered out of the seeing core and into the sky level. Since the flat field is computed on the basis of sky level, these gradients are seen as residuals in photometry. Radial photometry corrections for the $i^{\prime}$ and $z^{\prime}$ bandpasses were found by performing empirical fits to photometry residuals; they are applied to the $i^{\prime}$ and $z^{\prime}$ bandpass photometry. These "thinning" corrections reach a maximum of $\left[\Delta i^{\prime}, \Delta z^{\prime}\right] \sim[0.02,0.05] \mathrm{mag}$ at the field edges and $\sim[0.03$, $0.07]$ mag at the field corners.

\section{CLOUD DETECTION AND DATA VETO}

The "prototype cloud camera" (Hull et al. 1994) operating at APO utilizes a single-pixel cooled $10 \mu \mathrm{m}$ detector and scanning mirrors. The sky is scanned by two flat mirrors driven by stepper motors, followed by an off-axis hyperbolic mirror that images the sky onto a single channel HgCdTe photoconductive detector. ${ }^{9}$ The detector samples 300 times per scan, 300 scans per image, yielding an image with $300 \times 300$ pixels covering a $135 \times 135 \mathrm{deg}^{2}$ field with the 0.9 beam. Some typical images are shown in Figure 2. An image is completed in approximately 5 minutes. This is somewhat slow for real-time monitoring, but perfectly adequate for our purposes. This design was preferred over a solid-state array for reasons of cost, stability, and field of view. A disadvantage is the maintenance required by moving parts, but this has not been a serious drawback.

Experimentation showed that a simple and adequate method for detecting cloud cover is to compute the rms value of the sky within $45^{\circ}$ of zenith in each frame. This seems to be a simple and robust method, and it fails only in the case of unnaturally uniform cloud cover (this has never occurred). When the cloud-camera rms exceeds a predefined threshold for a period of time, that period is declared bad, and the data taken during that interval are ignored for

\footnotetext{
${ }^{9}$ For more information on the cloud camera, see http:// www.apo.nmsu.edu/telescopes/sdss/sdss.html.
} 

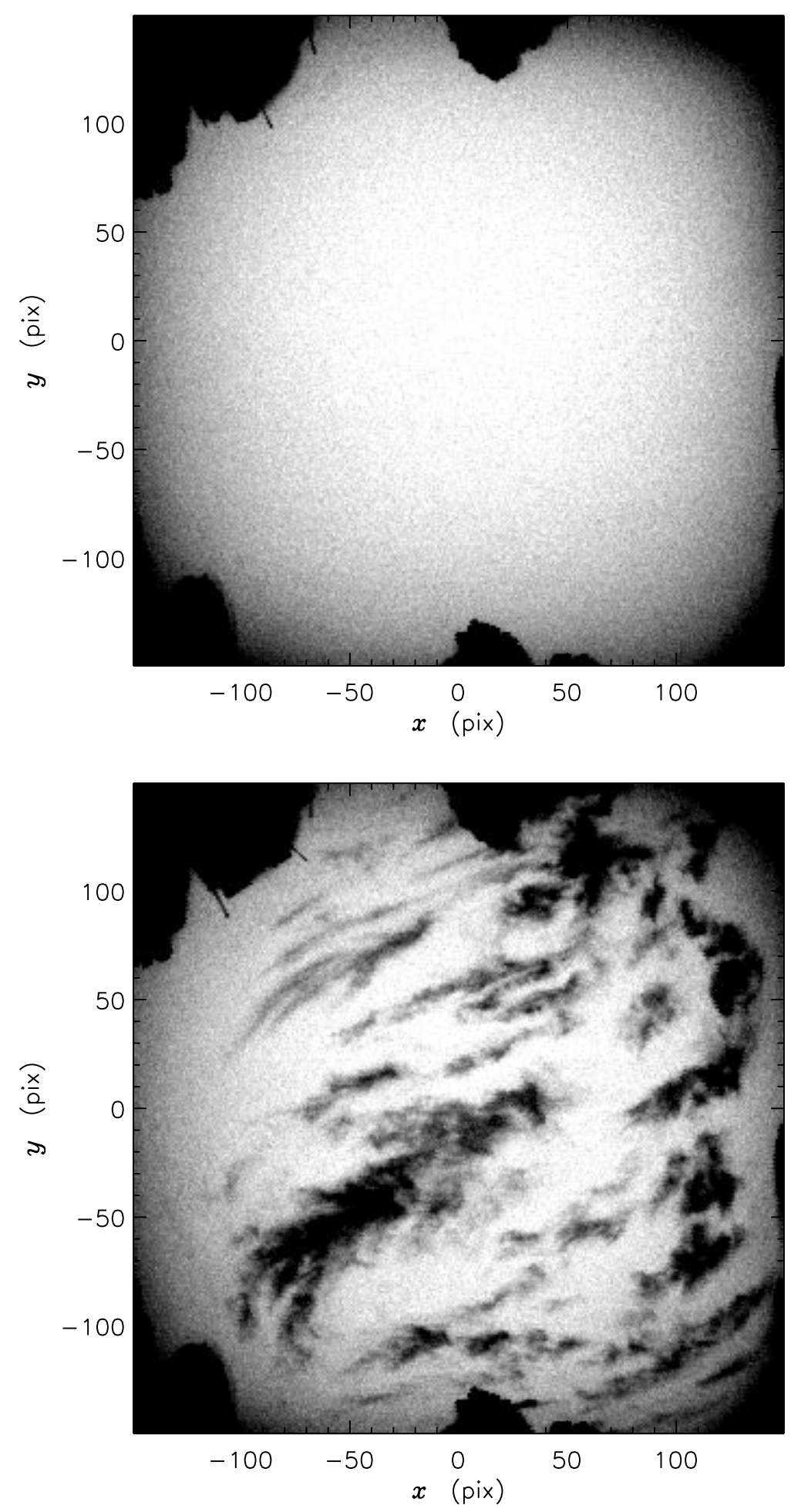

FIG. 2.-Two example cloud-camera images, taken at 05:24 UT on MJD 51997 (top), and at 05:45 UT on MJD 51997 (bottom). The images are displayed at the same stretch and are negatives (bright areas appear dark). The dark silhouettes around the edge are buildings and trees near the camera; the APO $3.5 \mathrm{~m}$ telescope is visible at upper left.

photometric parameter fitting. The bad interval is padded by 20 minutes on each end, so that even a single cloud appearing in one frame requires discarding at least 40 minutes of data. This is conservative, but it is more robust to set the cloud threshold high and reject significant time intervals than to make the threshold extremely low.

\section{PHOTOMETRIC SOLUTION}

Every time the PT completes a set of $u^{\prime}, g^{\prime}, r^{\prime}, i^{\prime}$, and $z^{\prime}$ exposures in a field, the robot compiles all the measurements made of photometric standard stars in that night and fits photometric parameters to all data not declared bad by 
the cloud-camera veto $(\S 8)$. The photometric equations used for the five bandpasses are

$$
\begin{aligned}
u_{\mathrm{inst}}^{\prime}= & u_{\mathrm{USNO}}+a_{u}+b_{u}(u-g)_{\mathrm{USNO}}+k_{u} X \\
& +c_{u}\left(X-X_{0}\right)\left[(u-g)_{\mathrm{USNO}}-(u-g)_{0}\right] \\
& +\dot{a}_{u}\left(t-t_{0}\right) \\
g_{\mathrm{inst}}^{\prime}= & g_{\mathrm{USNO}}+a_{g}+b_{g}(g-r)_{\mathrm{USNO}}+k_{g} X \\
& +c_{g}\left(X-X_{0}\right)\left[(g-r)_{\mathrm{USNO}}-(g-r)_{0}\right] \\
& +\dot{a}_{g}\left(t-t_{0}\right) \\
r_{\mathrm{inst}}^{\prime}= & r_{\mathrm{USNO}}+a_{r}+b_{r}(r-i)_{\mathrm{USNO}}+k_{r} X \\
& +c_{r}\left(X-X_{0}\right)\left[(r-i)_{\mathrm{USNO}}-(r-i)_{0}\right] \\
& +\dot{a}_{r}\left(t-t_{0}\right), \\
i_{\mathrm{inst}}^{\prime}= & i_{\mathrm{USNO}}+a_{i}+b_{i}(i-z)_{\mathrm{USNO}}+k_{i} X \\
& +c_{i}\left(X-X_{0}\right)\left[(i-z)_{\mathrm{USNO}}-(i-z)_{0}\right] \\
& +\dot{a}_{i}\left(t-t_{0}\right), \\
z_{\mathrm{inst}}^{\prime}= & z_{\mathrm{USNO}}+a_{z}+b_{z}(i-z)_{\mathrm{USNO}}+k_{z} X \\
& +c_{z}\left(X-X_{0}\right)\left[(i-z)_{\mathrm{USNO}}-(i-z)_{0}\right] \\
& +\dot{a}_{z}\left(t-t_{0}\right),
\end{aligned}
$$

where the terms subscripted "inst" symbolize instrumental magnitudes defined by

$$
m_{\mathrm{inst}} \equiv-2.5 \log _{10}\left(\frac{D N}{t_{\text {exp }}}\right)
$$

where $D N$ is the flux in raw counts in the corrected frame and $t_{\exp }$ is the exposure time; the terms subscripted "USNO" symbolize the magnitudes in the photometric standard-star catalog; $X$ symbolizes air mass; $t$ symbolizes time (UT); $X_{0}, t_{0}$, and the colors $(u-g)_{0}$, etc., symbolize fiducial air-mass, time, and colors (arbitrarily chosen but close to mean values), respectively; and the $a, b, k, c$, and $\dot{a}$ parameters are, in principle, free to vary. The system sensitivities are the $a_{i}$; the tiny differences in photometric systems between the USNO 40 inch $(1 \mathrm{~m})$ and PT bandpasses are captured by the color coefficients, $b_{i}$; the atmospheric extinction coefficients are the $k_{i}$; atmospheric extinction is a weak function of intrinsic stellar color parameterized by the $c_{i}$; and the $\dot{a}_{i}$ parameterize any small time evolution of the system during the night.

The above photometric equations are not strictly correct for an $A B$ system, because in the $A B$ system there is no guarantee that the colors of standard stars through two slightly different filter systems will agree at zero color; this agreement is assumed by the above equations. In an empirical system, such as the Vega relative magnitude system, a certain star, such as Vega (and stars like it), has zero color in all colors of all filter systems. The AB system is based on a hypothetical source with $f_{v}=$ const; there is no guarantee that a source with zero color in one filter system will have zero color in any other. The offsets must be computed theoretically, using models of CCD efficiencies, mirror reflectivities, atmospheric absorption spectra, and intrinsic stellar spectral energy distributions. We have ignored this (subtle) point, since it only leads to offsets in the sensitivity parameters $a_{i}$ and does not affect photometricity or atmospheric extinction assessments.
In practice, the $c$ parameters, in mag $\mathrm{mag}^{-1}$ per air mass, are always fixed at the theoretically derived values

$$
\begin{gathered}
c_{u}=-0.021, \quad c_{g}=-0.016, \\
c_{r}=-0.004, \quad c_{i}=+0.006, \\
c_{z}=+0.003 .
\end{gathered}
$$

The $\dot{a}$ parameters are allowed to be nonzero only in the next-day analysis (§ 11). Because the design specification on the PT did not require correct air-mass values in raw image headers, the air-mass values are computed on the fly from the right ascension, declination, UT, and location of the observatory (e.g., Smart 1977). The reference air mass and colors are chosen to be roughly the mean on a typical night of observing,

$$
\begin{gathered}
X_{0}=1.30 \text { airmass } \\
(u-g)_{0}=+1.42, \quad(g-r)_{0}=+1.11, \\
(r-i)_{0}=+0.48, \quad(i-z)_{0}=+0.35,
\end{gathered}
$$

where the colors are in magnitudes (in the AB system). In the next-day analysis, the reference time $t_{0}$ is set to be the mean UT of the observations.

In principle the color coefficients, $b_{i}$, could be determined globally and fixed once and for all. However, the PT filters have been shown to have some variation with time and with humidity (they are kept in a low-humidity environment with some variability), so the robot fits them independently every night. Freedom of the $b_{i}$ is allowed not because it is demanded by the data but rather because measurements of the $b_{i}$ over time are an important part of data and telescope quality monitoring. Typical color coefficient values (which measure differences between the USNO 40 inch $(1 \mathrm{~m})$ telescope and PT bandpasses) in mag $\mathrm{mag}^{-1}$ are

$$
\begin{gathered}
b_{u}=+0.048, \quad b_{g}=-0.004, \\
b_{r}=-0.028, \quad b_{i}=-0.053, \\
b_{z}=-0.031 .
\end{gathered}
$$

Quite a bit of experimentation went into the photometric equations. Inclusion of theoretically determined $c$ parameters minutely improves the fit, but on a typical night there are not enough data to determine the $c$ parameters empirically; the $c_{i}$ are held fixed. Terms proportional to products of time and air mass, again, are not well constrained from a single night's data; they are not included in the fit. To each bandpass a color is assigned; the choices have been made to use the most well-behaved "adjacent" colors. (The $u-g$ color is not used for the $g$ equation because the boundary between $u$ and $g$ is, by design, on the $4000 \AA$ break, making color transformations extremely sensitive to the precise properties of the filter curves.)

In the real-time analyses, the $a, b$, and $k$ parameters $(15$ total) are fitted to the counts from the aperture photometry (and USNO magnitudes, times, air masses, etc.) with a linear least-squares fitting routine, which iteratively removes $3.0 \sigma$ outliers and repeats its fit. A typical night at APO shows extinction coefficients

$$
\begin{gathered}
k_{u}=0.52, \quad k_{g}=0.19, \quad k_{r}=0.12, \\
k_{i}=0.08, \quad k_{z}=0.06,
\end{gathered}
$$

in mag air mass ${ }^{-1}$, with a factor of $\sim 1.5$ variations from night to night.

All photometric measurements are weighted equally in these fits, because the error budget appears to be dominated 
by systematic errors in the USNO catalog and sky subtraction; the primary errors are not from photon statistics. As observing continues and iterative improvement to the USNO catalog is made, we will be able to use a more sophisticated weighting model; we don't expect such improvements to change the values of our best-fit photometric parameters significantly. On a typical night, fiveband measurements are made of 20 to 50 standard stars in 10 to 15 standard-star fields.

\section{REAL-TIME OBSERVER FEEDBACK}

Every 10 minutes during the night (from 18:00 to 07:00, local time), the robot builds a WWW page in hypertext markup language ${ }^{10}$ (HTML), reporting on its most up-todate photometric solution. The WWW page shows the bestfit $a, b$ and $k$ parameter values, plots of residuals around the best photometric solution in the five bandpasses, and rms of the residuals in the five bandpasses. Parameters or rms values grossly out of specification are flagged in red. This feedback allows the observers to make real-time decisions about photometricity and to confirm expectations based on visual impressions of and $10 \mu \mathrm{m}$ camera data on atmospheric conditions. The WWW page also includes an excerpt from the observers' manually entered log file. This allows those monitoring the site remotely to compare the observers' and robot's photometricity judgments. Figures 3 and 4 show examples of the photometricity output on the WWW page on two typical nights. The WWW page also

${ }^{10}$ Information about HTML is available at http://www.w3.org/.

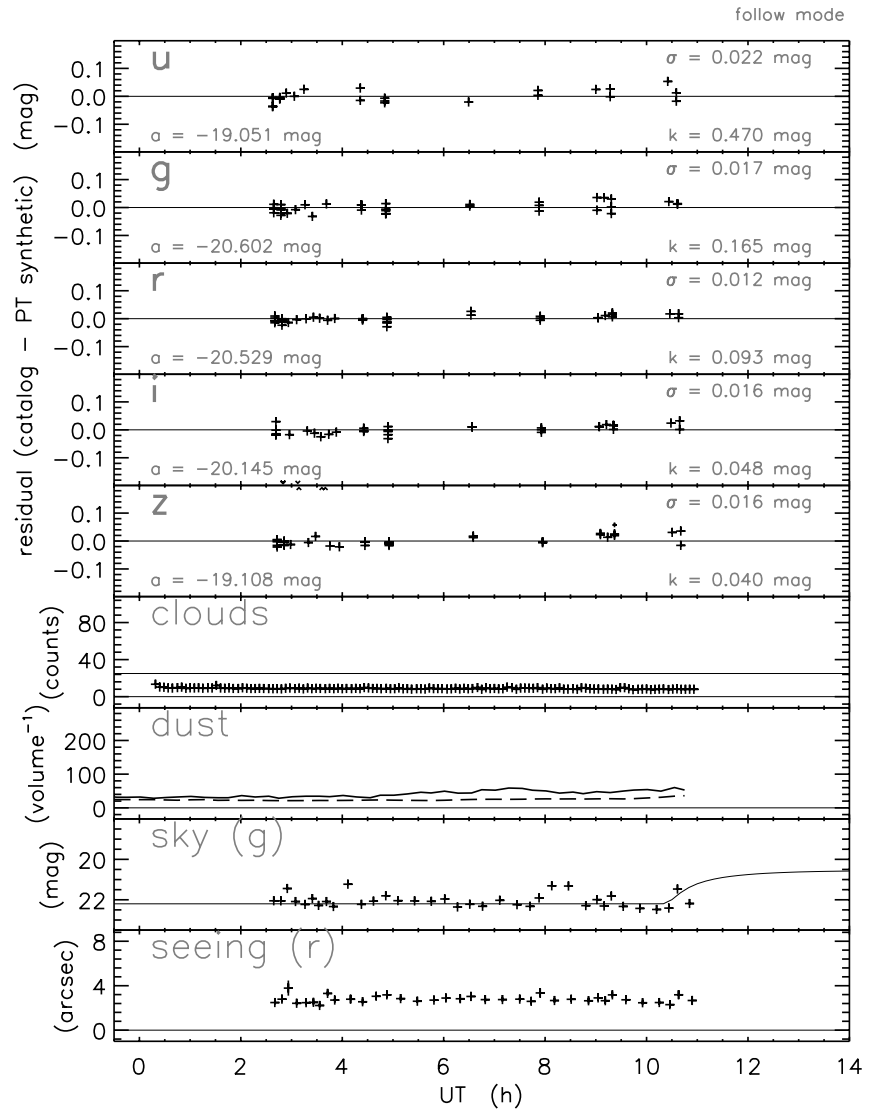

FIG. 3.- Real-time WWW output created at 11:00 UT on MJD 51987. This was one of the best nights of the year at APO.

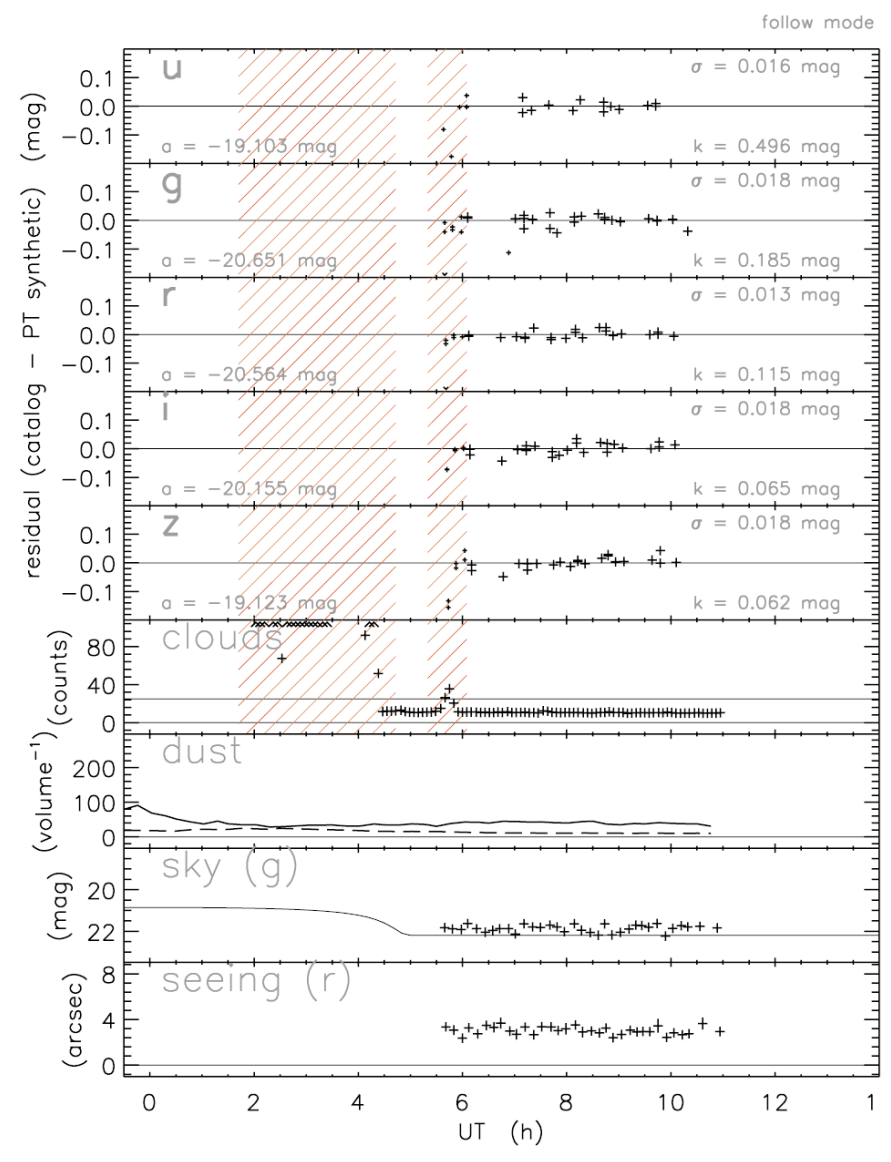

FIG. 4.-Real-time WWW output created at 11:00 UT on MJD 51997. The red hatched areas are time intervals vetoed by the cloud-camera analysis. Rejected points are shown with smaller symbols.

shows data from the APO weather, dust, and cloud monitors for comparison by the observers, as do Figures 3 and 4.

Since WWW pages in HTML are simply text files, they are trivially output by IDL. The figures on the WWW pages are output by IDL as PostScript files and then converted to a (crudely) antialiased pixel image with the UNIX command GHOSTSCRIPT ${ }^{11}$ and the UNIX PBMPLUS package. ${ }^{12}$

\section{NEXT-DAY ANALYSIS AND FEEDBACK}

At 07:00 (local time) each day, the robot begins its " nextday" analysis, in which all the data from the entire night (just ended) are rereduced and final decisions are made about photometricity and data acceptability. Bias frames taken during the night are identified by header keywords. The bias frames are averaged, with iterated outlier rejection, to make a bias image. Any pixels with values more than 10 $\sigma$ deviant from the mean pixel value in the bias image are flagged as bad in a bias mask image.

Dome and sky flat frames are identified by header keywords. These raw images are bias-subtracted using the bias image. Each bias-subtracted image is divided by its own mean, which is estimated with iterated outlier rejection. The

\footnotetext{
${ }^{11}$ Information about GHOSTSCRIPT is available from http:// www.gnu.org/.

12 Information about the PBMPLUS package is available from http:// www.acme.com/.
} 


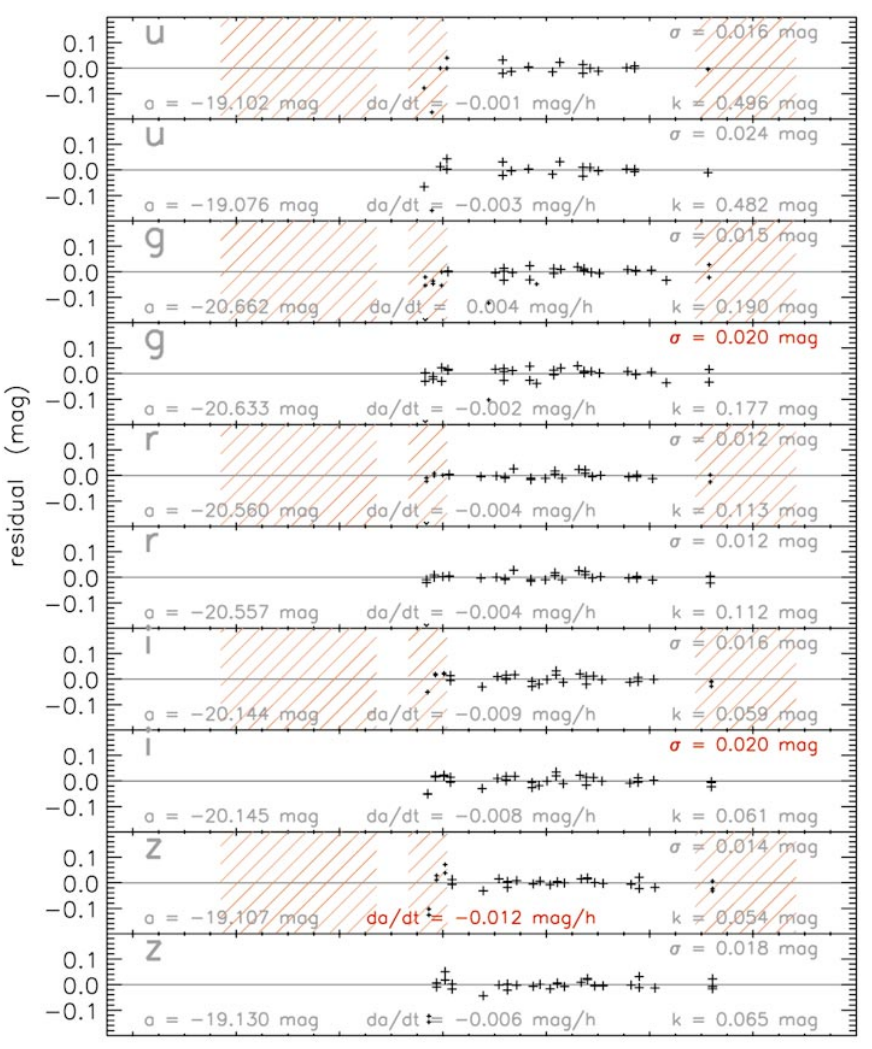

FIG. 5.-Comparison of final fits (i.e., including $\dot{a}$ terms) in the five bands, for MJD 51997, with and without the data vetoing by cloudcamera. The panels with the red cross-hatching have had data taken during cloudy periods removed from the fit. In most bands, the fit is improved when cloudy data are removed.

bias-subtracted and mean-divided flat frames are averaged together, again with iterated outlier rejection, to make five flat images, one for each bandpass. Any pixels with values more than $10 \sigma$ deviant from the mean are flagged as bad in flat mask images.

The $i$ - and $z$-bandpass images are affected by an interference fringing pattern, which is modeled as an additive distortion. Any $i$ - or $z$-bandpass image taken during the night with exposure time $t_{\text {exp }}>100 \mathrm{~s}$ is identified. These long-exposure frames are bias-subtracted and divided by the flat. Each is divided by its own mean, again estimated with iterated outlier rejection. These mean-divided frames are averaged together, again with iterated outlier rejection to make two fringe correction images, in the $i^{\prime}$ and $z^{\prime}$ bandpasses. Again, $10 \sigma$ outlier pixels are flagged as bad in fringe mask images.

Constant bias, flat, and fringe images are used for the entire night; there is no evidence with this system that there is time evolution in any of these. All the raw images from the night are bias-subtracted and flattened with these new same-night bias and flat images. The $i^{\prime}$ - and $z^{\prime}$-bandpass images are also fringe-corrected. Thus a new set of corrected frames is constructed for that night. The real-time astrometry solutions are reused in these new corrected frames. All photometry is remeasured in the new corrected frames.

A final photometric parameter fit is performed with the new measurements, again with removal of data declared bad by the cloud-camera veto, and with iterated outlier rejection. The only difference is that the time evolution terms, $\dot{a}$, are allowed to be nonzero.
Because we expect nonzero $\dot{a}$ terms to be caused by changing atmospheric conditions, we experimented with allowing time evolution in the extinction coefficients (e.g., $\dot{k}$ terms). Unfortunately, such terms involve nonlinear combinations of input data (time times air mass) and can be added without introducing biases only if there are also $\dot{a}$ terms; i.e., it is wrong in principle to include $\dot{k}$ terms without also adding $\dot{a}$ terms, especially in the face of iterated outlier rejection. We found that adding both $\dot{a}$ and $\dot{k}$ terms did not improve our fits relative to simply adding $\dot{a}$ terms, so we have not included $\dot{k}$ terms. With more frequent standardstar sampling, the time resolution of the system would be improved and $\dot{k}$ terms would, presumably, improve the fits.

The entire next-day reanalysis takes between 3 and 6 hours, depending on the amount of data. Much of the computer time is spent swapping processes in and out of virtual memory; with more efficient code or larger RAM the reanalysis time could be reduced to under 2 hours. The reanalysis would take 1 to 2 hours longer if it were necessary to repeat the astrometric solutions found in the real-time analysis.

At the end of the reanalysis the robot constructs a "final" WWW page, similar to the real-time feedback WWW page, but with the final photometric solution and residuals. $\mathrm{Pa}$ rameters grossly out of specification are shown in red. Also, the robot sends an email to an SDSS email archive and exploder, summarizing the final parameter values and $\mathrm{rms}$ residuals in the five bands.

For the robot's purposes, a night is declared "photometric" if the rms residual around the photometric solution (after iterated outlier rejection at the $3 \sigma$ level) in the $\left[u^{\prime}, g^{\prime}, r^{\prime}, i^{\prime}, z^{\prime}\right]$ bandpasses is less than $[0.03,0.02,0.02$, $0.02,0.03] \mathrm{mag}$. If the night is declared photometric, then the bias, flat, and fringe images and their associated pixel masks are declared "current," to be used for the real-time analysis of the following nights.

Figure 5 shows the final fits for an example night, with and without the inclusion of the veto of data taken during cloudy periods. This is a night that would have been declared marginally nonphotometric without the cloudcamera veto but became photometric when the cloudy periods were removed.

\section{ARCHIVING}

All raw PT data are saved in a tape archive at Fermi National Accelerator Laboratory (FNAL). In addition, about one year's worth of the most recent data are archived on the robot machine itself, on a pair of large disk drives. The photometric parameters from each photometric night are kept in a FITS binary table (Cotton, Tody, \& Pence 1995), labeled by observation date. These parameter files are mirrored in directories at FNAL and elsewhere (D. W. H.'s laptop, for example). Because the astrometric solutions are somewhat time-consuming, the astrometric headers for all the corrected frames are also saved on disk on the robot machine and mirrored, along with each night's bias, flat, and fringe images. Because the astrometric header information is all saved, along with the bias, flat, and fringe images, the corrected frames can be reconstructed trivially and quickly from the raw data.

\section{SCIENTIFIC OUTPUT}

The archived output data from the photometricity monitor robot are not just useful for verifying and analyzing 


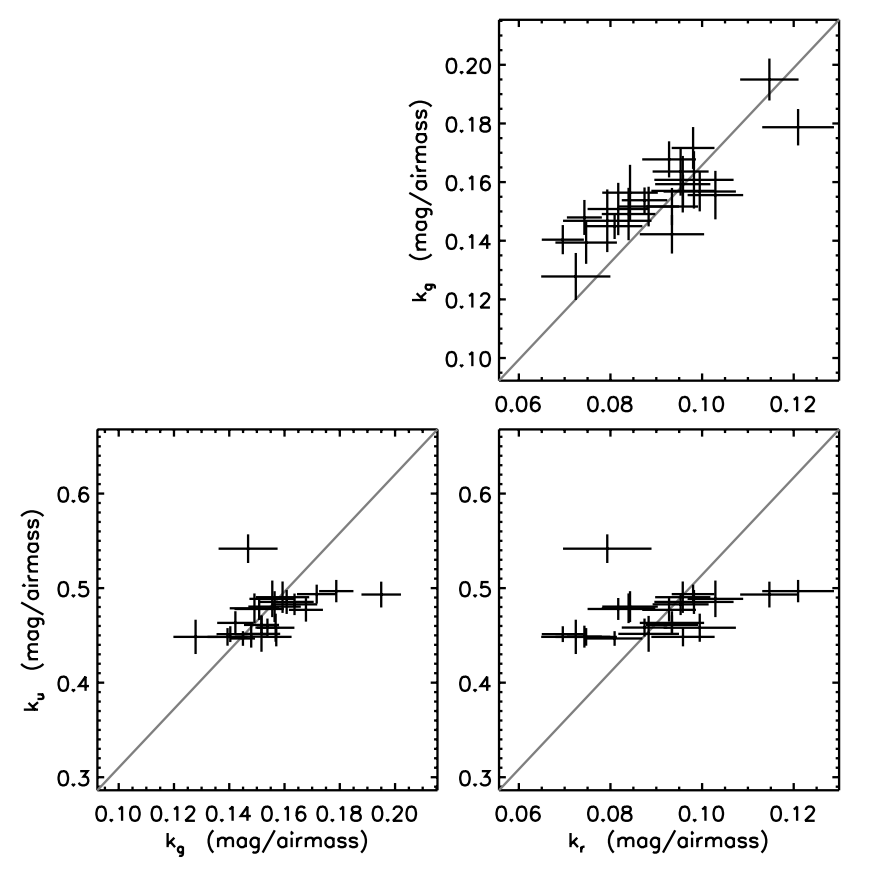

FIG. 6.-Atmospheric extinction coefficients $\left[k_{u}, k_{g}, k_{r}\right]$ in the $\left[u^{\prime}, g^{\prime}, r^{\prime}\right]$ bandpasses, for most photometric nights between MJD 51840 and 51970, plotted against one another. The diagonal lines show the relationship $\left(k_{u} \propto k_{g} \propto k_{r}\right)$ expected if atmospheric extinction is due to a single atmospheric component.

contemporaneous data from the observatory. They contain a wealth of scientific data useful for analyzing long-term behavior and pathologies of the site, the hardware, and the standard-star catalog. Many of these analyses will be performed and presented elsewhere, as the site monitoring data builds up.

As an example, Figure 6 shows the atmospheric extinction coefficients $\left[k_{u}, k_{g}, k_{r}\right]$ plotted against one another, for most of the photometric nights in roughly one-third of a year. This figure shows the variability in the extinction coefficients, as well as their covariance. If the variability in the extinction is due to varying optical depth of a single component of absorbing material, the covariances should fall along the diagonal lines (i.e., $k_{u} \propto k_{g} \propto k_{r}$ ). Although the $k_{g}$ versus $k_{r}$ plot is consistent with this assumption, the $k_{u}$ versus $k_{r}$ plot appears not to be. Perhaps not surprisingly, atmospheric extinction must be caused by multiple atmospheric components. It is at least slightly surprising to us that the variability in $u$-bandpass extinction is smaller than would be predicted from a one-component assumption and the variability in the $r$-bandpass extinction.

\section{COMMENTS}

The success of this ongoing project shows that robust, hands-off, real-time, and next-day photometricity assessment and atmospheric extinction measurement is possible. There is much lore about photometricity, site variability, and precise measurement in astronomy. Most of this lore can be given an empirical basis with a simple system such as the one described in this paper.

It is worth emphasizing that the observing hardware used by the robot is not dedicated to the robot's site monitoring tasks. The PT is being used to calibrate SDSS data; it spends only about one-third of its time taking the obser- vations of cataloged standards that are used for photometricity and extinction measurements. This shows that a robot of this type could, with straightforward (if not trivial) adjustment, be made to "piggyback" on almost any observational program, provided that some fraction of the data is multiband imaging of photometric standard stars. The robot does not rely on the images having accurate astrometric header information or accurate text descriptions or log entries; it finds standard stars in a robust, hands-off manner. Many observatories could install a robot of this type with no hardware cost whatsoever! A site with no appropriate imaging program on which the robot could "piggyback" could install a small telescope with a good robotic control system, a CCD and filter wheel, and the robot software described here at fairly low cost. The telescope need only be large enough to obtain good photometric measurements of some appropriate set of standard stars. All the costs associated with such a system are going down as small robotic telescopes are becoming more common (e.g., Akerlof et al. 1999; Strassmeier et al. 2000; Querci \& Querci 2000).

The robot works adequately without input from the 10 $\mu \mathrm{m}$ cloud camera, as long as data are not taken during cloudy periods or as long as the observers can mark, in some way accessible to the robot, cloudy data. On the other hand, $10 \mu \mathrm{m}$ pixel array cameras, with no moving parts (unlike the prototype camera working at APO) are now extremely inexpensive and would be easy to install and use at any observatory.

The robot system was developed and implemented in a period of about nine months. It has been a very robust tool for the SDSS observers. The rapid development and robust operation can be ascribed to a number of factors: The robot design philosophy has always been to make every aspect of the robot's operation as straightforward as possible. We have added sophistication to the robot's behavior only as it has been demanded by the data. The IDL data analysis language has primitives useful for astronomy and it operates on a wide range of platforms and operating systems. Perhaps above all, the PT is a stable, robust telescope (Uomoto et al. 2001).

Without objective, well-understood site monitoring such as that provided by this simple robot, analyses of archived and queue-observing data will always be subject to some suspicion. At APO, the visual monitor robot has been a very inexpensive and effective tool for building confidence in observer decisions and for providing feedback to data analysis. With this robot operating, APO has better monitoring of site conditions and data quality than most existing or even planned observatories.

Comments, suggestions, data, computer code, bug reports, and hardware maintenance were provided generously by Bill Boroski, Jon Brinkmann, Scott Burles, Bing Chen, Daniel Eisenstein, Masataka Fukugita, Steve Kent, Jill Knapp, Wayne Landsman, Brian Lee, Craig Loomis, Robert Lupton, Pete Newman, Eric Neilsen, Kurt Ruthsmandorfer, Don Schneider, Steph Snedden, Chris Stoughton, Michael Strauss, Douglas Tucker, Alan Uomoto, Brian Yanny, Don York, our anonymous referee, and the entire staff of the Apache Point Observatory.

The Sloan Digital Sky Survey (SDSS) is a joint project of the University of Chicago, Fermilab, the Institute for Advanced Study, the Japan Participation Group, the 
Johns Hopkins University, the Max-Planck-Institute for Astronomy, the Max-Planck-Institute for Astrophysics, New Mexico State University, Princeton University, the United States Naval Observatory, and the University of Washington. Apache Point Observatory, site of the SDSS telescopes, is operated by the Astrophysical Research Consortium.

Funding for the project has been provided by the Alfred P. Sloan Foundation, the SDSS member institutions, the National Aeronautics and Space Administration, the
National Science Foundation, the US Department of Energy, the Japanese Monbukagakusho, and the Max Planck Society. ${ }^{13}$

Partial support for D. P. F. has been provided by NASA. This research made use of the NASA Astrophysics Data System.

13 The SDSS Web site is http://www.sdss.org/.
Adelman, S. J., Dukes, R. J., \& Adelman, C. J., eds. 1992, ASP Conf. Ser. 28, Automated Telescopes for Photometry and Imaging (San Francisco: ASP)

Akerlof, C., et al. 1999, Nature, 398, 400

Baruch, J. E., \& da Luz Vieira, J. 1993, Proc. SPIE, 1945, 488

Boroson, T., Davies, J., \& Robson, I., eds. 1996, ASP Conf. Ser. 87, New Observing Modes for the Next Century (San Francisco: ASP)

Boroson, T. A., Harmer, D. L., Saha, A., Smith, P. S., Willmarth, D. W., \& Silva, D. R. 1998, Proc. SPIE, 3349, 41

Brunner, R. J., Djorgovski, S. G., \& Szalay, A. S., eds. 2001, ASP Conf. Ser. 225, Virtual Observatories of the Future (San Francisco: ASP)

Calabretta, M., \& Greisen, E. W. 2000, in ASP Conf. Ser. 216, Astronomical Data Analysis Software and Systems IX, ed. N. Manset, C. Veillet, \& D. Crabtree (San Francisco: ASP), 571

Castro-Tirado, A. J., et al. 1999, A\&AS, 138, 583

Cotton, W. D., Tody, D., \& Pence, W. D. 1995, A\&AS, 113, 159

Filippenko, A. V., ed. 1992, ASP Conf. Ser. 34, Robotic Telescopes in the 1990s (San Francisco: ASP)

Fukugita, M., Ichikawa, T., Gunn, J. E., Doi, M., Shimasaku, K., \& Schneider, D. P. 1996, AJ, 111, 1748

Garzon, F., \& Rozas, M. 1998, Proc. SPIE, 3349, 319

Hull, C. L., Limmongkol, S., \& Siegmund, W. A. 1994, Proc. SPIE, 2199, 852

Landolt, A. U. 1992, AJ, 104, 340

Lupton, R. H., Gunn, J. E., Ivezic, Z., Knapp, G. R., Kent, S., \& Yasuda, N. 2001a, in ASP Conf. Ser. 238, Astronomical Data Analysis Software and Systems X, ed. F. R. Harnden, Jr., F. A. Primini, \& H. E. Payne (San Francisco: ASP)

Lupton, R. H., et al. 2001b, in preparation

Manset, N., Veillet, C., \& Crabtree, D., eds. 2000, ASP Conf. Ser. 216, Astronomical Data Analysis Software and Systems IX (San Francisco: ASP)
Massey, P., Guerrieri, M., \& Joyce, R. 2000, NewA, 5, 25

Mehringer, D. M., Plante, R. L., \& Roberts, D. A., eds. 1999, ASP Conf Ser. 172, Astronomical Data Analysis Software and Systems VIII (San Francisco: ASP)

Monet, D., et al. 1998, The USNO-SA2.0 Catalog (Washington: US Naval Obs.)

Oke, J. B., \& Gunn, J. E. 1983, ApJ, 266, 713

Querci, F. R., \& Querci, M. 2000, Ap\&SS, 273, 257

Russell, J. L., Lasker, B. M., McLean, B. J., Sturch, C. R., \& Jenkner, H. 1990, AJ, 99, 2059

Smart, W. M. 1977, Textbook on Spherical Astronomy (6th ed.; Cambridge: Cambridge Univ. Press)

Smith, J. A., et al. 2001, in preparation

Stetson, P. B. 1987, PASP, 99, 191

. 1992, in ASP Conf. Ser. 25, Astronomical Data Analysis Software and Systems I, ed. D. M. Worrall, C. Biemesderfer, \& J. Barnes (San Francisco: ASP), 297

Strassmeier, K. G., Granzer, T., Boyd, L. J., \& Epand, D. H. 2000, Proc. SPIE, 4011, 157

Tilanus, R. P. J. 2000, in ASP Conf. Ser. 216, Astronomical Data Analysis Software and Systems IX, ed. N. Manset, C. Veillet, \& D. Crabtree (San Francisco: ASP), 101

Uomoto, A., et al. 2001, in preparation

Valdes, F. G., Campusano, L. E., Velasquez, J. D., \& Stetson, P. B. 1995, PASP, 107, 1119

Wells, D. C., Greisen, E. W., \& Harten, R. H. 1981, A\&AS, 44, 363

York, D. G., et al. 2000, AJ, 120, 1579 